\title{
Thermo-responsive liquid marbles
}

\author{
Polymer Journal (2014) 46, 145-148; doi:10.1038/pj.2013.84; published online 23 October 2013
}

Aussillous and Quéré used the term liquid marbles to refer to non-stick droplets coated with micro- or nano-scale hydrophobic powder particles. $^{1}$ A liquid droplet is encapsulated by a hydrophobic powder at the liquid-air interface. Liquid marbles have been prepared using powders such as lycopodium powder, $^{2}$ silica, ${ }^{3}$ carbon black, ${ }^{4}$ poly[2-(perfluorooctyl)ethyl acrylate $],{ }^{5}$ polytetrafluoroethylene, ${ }^{6}$ polyvinylidene fluoride, ${ }^{7}$ aerogels, ${ }^{8}$ graphite ${ }^{9}$ and others. ${ }^{10-12}$ The surface wettability of the powder is an important parameter for the preparation of liquid marbles. The hydrophobic liquid marble shell prevents direct contact between the liquid core and surfaces outside the marble shell. Liquid marbles are spherical and retain a stable shape while on the surface of a solid or liquid. Liquid marbles have shown particular promise for applications in water surface pollution detection, ${ }^{13}$ gas detection $^{14}$ and microreactors. ${ }^{15}$

Recent reports have described various stimulus-responsive liquid marbles with stability that can be controlled by external stimuli. For example, liquid marbles stabilized by particles containing $\mathrm{pH}$-responsive polymers such as deprotonated hydrophobic poly ((2-dimethylamino)ethyl methacrylate $)^{16}$ and poly(2-vinylpyridine) (P2VP), ${ }^{17}$ which can float on the surface of water, disintegrate upon the addition of acid to the bulk water because the particle surface becomes hydrophilic owing to the protonation of the $\mathrm{pH}$-responsive polymers. Wang et al. ${ }^{18}$ reported the preparation of magnet- and ultraviolet (UV)-responsive liquid marbles using $\mathrm{Fe}_{3} \mathrm{O}_{4} / \mathrm{SiO}_{2}$ particles with a pH-responsive block copolymer containing P2VP and a photo acid generator. The movement of these particles can be controlled by an external magnetic field. Upon UV irradiation, the photo acid generator-generated acid, which induces ionization of the P2VP chains on the particle surface, leads to the disintegration of the liquid marble. Recently, UV-responsive liquid marbles were prepared using a water droplet covered with photochromic spiropyran powder. ${ }^{19}$ These UV-responsive liquid marbles disintegrated upon UV irradiation because the hydrophobic spiropyran was transformed into hydrophilic merocyanine, which has a betaine structure. The development of stimulus-responsive liquid marbles may expand their area of application. It is interesting to investigate new stimulus-responsive liquid marbles, including thermally responsive liquid marbles.

An aqueous solution of poly( $N$-isopropylacrylamide) (PNIPAM) undergoes a thermally reversible phase separation. ${ }^{20}$ At room temperature $\left(<32^{\circ} \mathrm{C}\right)$, PNIPAM is hydrophilic and dissolves in water, adopting a random coil conformation because of hydrogen bonding between the pendant amide groups and water molecules. However, PNIPAM separates from the aqueous phase when heated to temperatures higher than $32{ }^{\circ} \mathrm{C}$, a lower critical solution temperature (LCST). This finding indicates that PNIPAM powder may exhibit hydrophilic properties below the LCST and may become hydrophobic above the LCST. The LCST for PNIPAM is influenced by the type and concentration of salts added to the aqueous solution. ${ }^{21,22}$

In the current study, thermo-responsive liquid marbles (Figure 1) were prepared using PNIPAM powder. It was difficult to maintain precise control of the temperature for the liquid marbles because room temperature $\left(\sim 22^{\circ} \mathrm{C}\right)$ was below the LCST. To simplify the experiments, a stable liquid marble floating on the surface of bulk water in a Petri dish disintegrated when the temperature of bulk water decreased below room temperature. This strategy involved the preparation of stable liquid marbles using PNIPAM powder at room temperature followed by a decrease in the LCST below room temperature. To decrease the LCST, salt $\left(\mathrm{Na}_{2} \mathrm{SO}_{4}\right)$ was added to the water droplet and the bulk water.

\section{EXPERIMENTAL PROCEDURE}

NIPAM from Kohjin Holdings Co. (Tokyo, Japan) was purified by recrystallization from a mixture of benzene and $n$-hexane (3/7, v/v). N,N-dimethylformamide (DMF, 99.0\%) was dried over $4 \AA$ molecular sieves and purified by distillation under reduced pressure. 2,2'-Azobis(isobutyronitrile) (98\%) from Wako Pure Chemical (Tokyo, Japan) was recrystallized from methanol. Sodium sulfate $\left(\mathrm{Na}_{2} \mathrm{SO}_{4},>99.0 \%\right)$, iron(III) nitrate nonahydrate $\left(\mathrm{Fe}\left(\mathrm{NO}_{3}\right)_{3} \cdot 9 \mathrm{H}_{2} \mathrm{O}, \quad 99.9 \%\right)$, potassium hexacyanoferrate(II) trihydrate $\left(\mathrm{K}_{4}\left[\mathrm{Fe}(\mathrm{CN})_{6}\right] \cdot 3 \mathrm{H}_{2} \mathrm{O}, 99.5 \%\right)$ and safranin were purchased from Wako Pure Chemical and were used as received.

PNIPAM was prepared by conventional free radical polymerization as follows. NIPAM (3.00 g, $26.5 \mathrm{mmol})$ and 2,2'-azobi$\mathrm{s}$ (isobutyronitrile) $\quad(21.7 \mathrm{mg}, \quad 0.133 \mathrm{mmol})$ were dissolved in DMF $(15 \mathrm{ml})$. The solution was heated at $60^{\circ} \mathrm{C}$ for $16 \mathrm{~h}$ under an $\mathrm{Ar}$ atmosphere. After reaction, the reaction mixture was poured into a large excess of diethyl ether/ $n$-hexane $(5 / 5, \mathrm{v} / \mathrm{v})$ to precipitate the polymer. The PNIPAM was obtained as a white powder and was dried under vacuum at $60{ }^{\circ} \mathrm{C}$ for one day $(2.59 \mathrm{~g}, 86.3 \%)$. The chemical structure was confirmed by ${ }^{1} \mathrm{H}$ nuclear magnetic resonance (Supplementary Figure S1). The number-average molecular weight $\left(M_{\mathrm{n}}\right)$ and molecular weight distribution $\left(M_{\mathrm{w}} / M_{\mathrm{n}}\right)$ were $1.93 \times 10^{4}$ and 3.80 , respectively, estimated from gel-permeation chromatography (Supplementary Figure S2).

${ }^{1} \mathrm{H}$ Nuclear magnetic resonance spectra were obtained using a DRX-500 spectrometer (Bruker Biospin Co., Kanagawa, Japan). The gel-permeation chromatography measurements were performed using an instrument equipped with an RI8021 refractive index detector (Tosoh Co., Tokyo, Japan) 
and two Shodex LF-804 polystyrene mixed gel columns (Showa Denko K.K., Tokyo, Japan; bead size $=7 \mu \mathrm{m}$, pore size $=20$ $200 \AA$ ) operated at $40^{\circ} \mathrm{C}$ under a flow rate of $0.5 \mathrm{ml} \mathrm{min}^{-1}$. A DMF solution containing 10-mM LiBr was used as the eluent. $M_{\mathrm{n}}$ and $M_{\mathrm{w}} / M_{\mathrm{n}}$ were calibrated using standard polystyrene samples. The values of $\% T$ for PNIPAM aqueous solutions at a constant polymer concentration $\left(C_{\mathrm{p}}\right)$ of $1.0 \mathrm{gl}^{-1}$ were measured using a V-530 spectrophotometer (Jasco Co., Tokyo, Japan) with a $1.0 \mathrm{~cm}$ path length quartz cell at various temperatures. The temperature was adjusted from 0 to $40{ }^{\circ} \mathrm{C}$ at a heating rate of $1.0^{\circ} \mathrm{C} \mathrm{min}^{-1}$ using an ETC-505T thermostat system (Jasco Co.). The fine structure of the powder was observed using a Keyence Co. (Tokyo, Japan) VE-9800 scanning electron microscope (SEM) at $12 \mathrm{kV}$. Samples were prepared for SEM by placing powder directly onto electrically conductive tape. The sample was treated by Pt sputtering using a Quick Coater Sc-701 MKII (Sanyu Electron Co., Tokyo, Japan). The surface temperature of the liquid marbles was monitored using a CPA8000 infrared camera (Chino Co., Tokyo, Japan). Digital photographs of the liquid marbles were obtained using an EOS Kiss X5 digital camera (Canon Inc., Tokyo, Japan).

\section{RESULTS AND DISCUSSION}

Liquid marbles can be prepared using PNIPAM powder and hot pure water at $60^{\circ} \mathrm{C}$, which is above the LCST. The marbles were prepared as follows. First a ground PNIPAM powder bed was prepared in a polytetrafluoroethylene dish. A 10- $\mu$ l hot water droplet was deposited on the PNIPAM powder bed using a syringe. Gentle rolling of the hot water droplet on the powder bed led to the complete encapsulation of the droplet by the powder, resulting in a liquid marble. When the temperature was maintained above $60{ }^{\circ} \mathrm{C}$, the liquid marbles were stable and remained intact after transfer onto a glass slide or onto the surface of bulk hot water at $60^{\circ} \mathrm{C}$. To decrease the LCST, $\mathrm{Na}_{2} \mathrm{SO}_{4}$ salt was added to the water droplet and the bulk water. The LCST of PNIPAM depends strongly on the type and concentration of added salt. In general, the LCST for PNIPAM in water decreased with increasing salt concentration because of salting out effects. Supplementary Figure S3 shows the percentage transmittance $(\% T)$ at $600 \mathrm{~nm}$ for aqueous PNIPAM solutions as a function of temperature from 0 to $40^{\circ} \mathrm{C}$ in the presence of varying concentrations of $\mathrm{Na}_{2} \mathrm{SO}_{4}$. Without $\mathrm{Na}_{2} \mathrm{SO}_{4}$, the LCST for PNIPAM in pure water was $32{ }^{\circ} \mathrm{C}$, which is similar to the values reported in the literature. ${ }^{20}$ The LCST decreased with increasing $\mathrm{Na}_{2} \mathrm{SO}_{4}$ concentration. The LCST for PNIPAM was $8{ }^{\circ} \mathrm{C}$ in the presence of $\mathrm{Na}_{2} \mathrm{SO}_{4}(0.5 \mathrm{M})$. This observation suggests that the wettability of the PNIPAM powder with aqueous $\mathrm{Na}_{2} \mathrm{SO}_{4}(0.5 \mathrm{M})$ solutions was low at room temperature.

A liquid marble was prepared using aqueous $0.5 \mathrm{M} \mathrm{Na} \mathrm{Na}_{4}$ covered with ground PNIPAM powder (Figure 2). The liquid marble was prepared at room temperature. The liquid marbles exhibited significant surface roughness, suggesting that they were coated with irregular powder clusters. The PNIPAM powder may trap air, thus resulting in a Cassie-Baxter wetting regime at the powder-liquid interface inside the liquid marble. ${ }^{4}$ The shape of the irregular PNIPAM powder clusters, which is nonuniform and indeterminate, can be observed by SEM (Supplementary Figure S4).

The liquid marbles were prepared by rolling a $10-\mu \mathrm{l}$ droplet of aqueous $0.5 \mathrm{M} \mathrm{Na} \mathrm{NO}_{4}$ containing safranin dye over a ground PNIPAM powder bed, which was then transferred

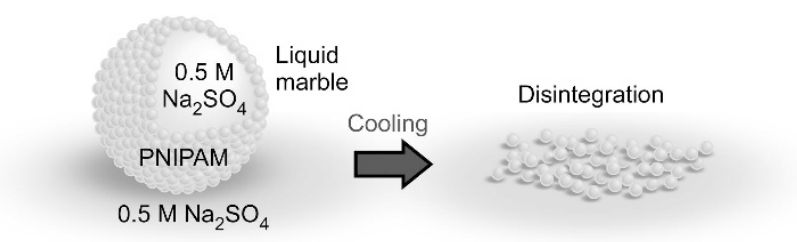

Figure 1 Conceptual illustration of a liquid marble consisting of a droplet of $0.5 \mathrm{M} \mathrm{Na} \mathrm{SO}_{4}$ aqueous solution covered with poly $(\mathrm{N}$-isopropylacrylamide) (PNIPAM) floated on the surface of aqueous $0.5 \mathrm{M}$ $\mathrm{Na}_{2} \mathrm{SO}_{4}$, followed by the disintegration of the liquid marble by cooling. A full color version of this figure is available at Polymer Journal online.

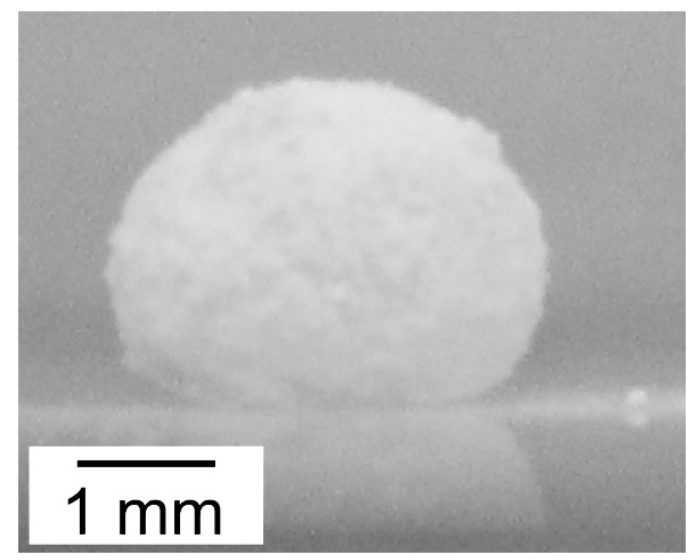

Figure $2 \mathrm{~A}$ digital photograph of a liquid marble containing aqueous $0.5 \mathrm{M} \mathrm{Na} \mathrm{SO}_{4}(10 \mu \mathrm{l})$ covered by poly( $\mathrm{N}$-isopropylacrylamide) powder on a glass substrate at room temperature. A full color version of this figure is available at Polymer Journal online.


Figure 3 Digital photographs of a floatable liquid marble made from an aqueous safranin dye solution containing $0.5 \mathrm{M} \mathrm{Na} \mathrm{SO}_{4}$ covered with poly ( $\mathrm{N}$-isopropylacrylamide) powder on bulk water containing $0.5 \mathrm{M} \mathrm{Na}_{2} \mathrm{SO}_{4}$ (a) at room temperature and (b) after cooling to $0{ }^{\circ} \mathrm{C}$ using an ice bath for $5 \mathrm{~min}$. A full color version of this figure is available at Polymer Journal online. 
to the surface of bulk water containing $0.5 \mathrm{M}$ $\mathrm{Na}_{2} \mathrm{SO}_{4}$ at room temperature (Figure 3 ). The bulk water was not colored when the liquid marble containing safranin dye was stable on the surface. However, when bulk water was cooled using an ice bath, the liquid marble disintegrated and leaked safranin dye from the interior of the liquid marble to the bulk water because the PNIPAM powder that formed the liquid marble dissolved in the water below the LCST. A video of the thermoresponsive behavior of the liquid marble is provided as Supplementary Video 1.
Figure $4 \mathrm{a}$ reports the disintegration temperatures of liquid marbles formed from PNIPAM powder and water droplets containing various $\mathrm{Na}_{2} \mathrm{SO}_{4}$ concentrations floated on bulk water containing the same concentration of $\mathrm{Na}_{2} \mathrm{SO}_{4}$ as in the liquid marble. The surface temperatures of the liquid marble were monitored using an infrared camera (Supplementary Figure S5). Measurements were performed five times for each $\mathrm{Na}_{2} \mathrm{SO}_{4}$ concentration, and the average destabilization temperatures were plotted against $\mathrm{Na}_{2} \mathrm{SO}_{4}$ concentration (Figure $4 \mathrm{~b}$ ). The disintegration
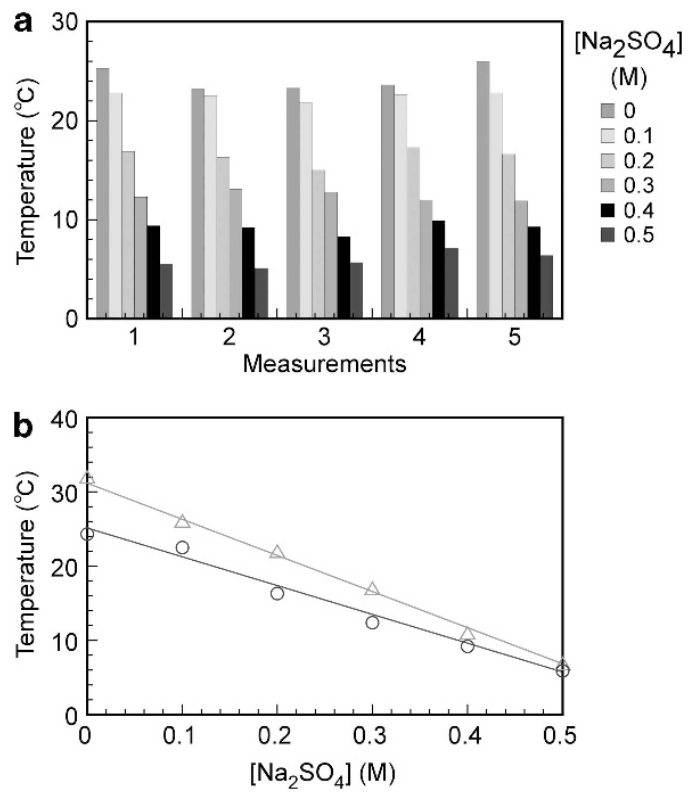

Figure 4 (a) Disintegration temperatures of the liquid marble prepared from poly $(N$ isopropylacrylamide) (PNIPAM) powder and $10 \mu \mathrm{l}$ water containing various concentrations of $\mathrm{Na}_{2} \mathrm{SO}_{4}$ floated on bulk water with the same $\mathrm{Na}_{2} \mathrm{SO}_{4}$ concentration as in the liquid marble. Measurements were performed five times for each $\mathrm{Na}_{2} \mathrm{SO}_{4}$ concentration. (b) The effect of $\mathrm{Na}_{2} \mathrm{SO}_{4}$ concentration on the disintegration temperature of the liquid marble $(O)$ and on the lower critical solution temperature of the aqueous PNIPAM solution $(\triangle)$. A full color version of this figure is available at Polymer Journal online.
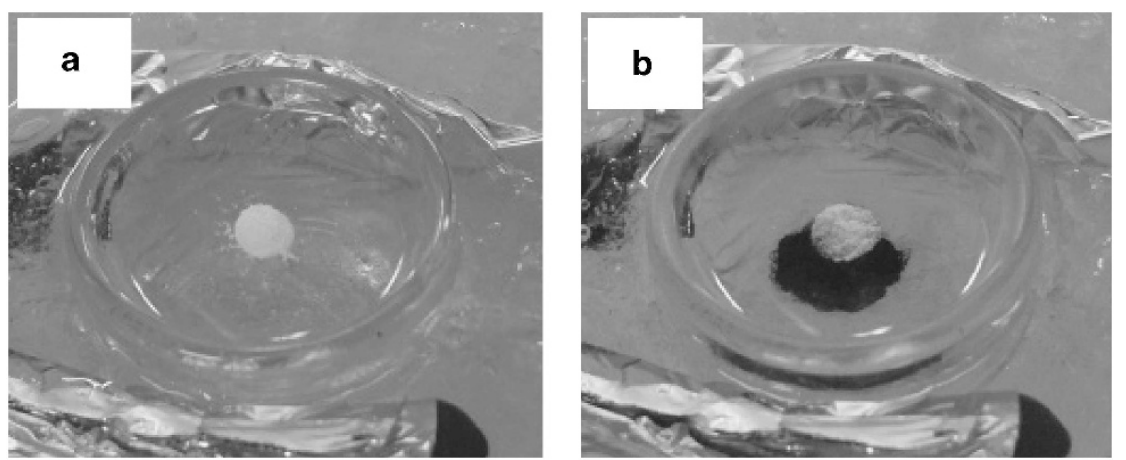

Figure 5 Digital photographs of a temperature-triggered chemical reaction using a $\operatorname{poly}(N$ isopropylacrylamide) (PNIPAM) liquid marble consisting of a water droplet containing $0.5 \mathrm{~m} \mathrm{Na}_{2} \mathrm{SO}_{4}$ and $0.2 \mathrm{M} \mathrm{K}_{4}\left[\mathrm{Fe}(\mathrm{CN})_{6}\right]$ covered with PNIPAM floating on bulk water containing $0.5 \mathrm{M} \mathrm{Na}_{2} \mathrm{SO}_{4}$ and $0.01 \mathrm{~m} \mathrm{Fe}\left(\mathrm{NO}_{3}\right)_{3}$ (a) before and (b) after cooling in an ice bath. A full color version of this figure is available at Polymer Journal online.

temperature of the liquid marble decreased with the increasing $\mathrm{Na}_{2} \mathrm{SO}_{4}$ concentration. At an $\mathrm{Na}_{2} \mathrm{SO}_{4}$ concentration of $0.5 \mathrm{M}$, the LCST value decreased to $6{ }^{\circ} \mathrm{C}$. The stability of the liquid marbles was enhanced above room temperature, indicating that PNIPAM powder was sufficiently hydrophobic to adsorb strongly at the air-liquid interface under these conditions. In contrast, liquid marbles containing $0.5 \mathrm{M} \mathrm{Na}_{2} \mathrm{SO}_{4}$ were always unstable and disintegrated when the surface temperature of the liquid marble decreased below $6{ }^{\circ} \mathrm{C}$, because of the dissolution caused by hydrated PNIPAM, followed by the disintegration of the liquid marble. The liquid marble formed from PNIPAM powder and a water droplet containing $0.5 \mathrm{M} \mathrm{Na}_{2} \mathrm{SO}_{4}$ remained stable for longer than a day at room temperature under humid conditions. Therefore, the liquid marble of an aqueous $\mathrm{Na}_{2} \mathrm{SO}_{4}$ solution coated with PNIPAM powder placed on bulk water containing $\mathrm{Na}_{2} \mathrm{SO}_{4}$ disintegrated in response to cooling. The disintegration temperature of the liquid marble was close to the LCST of PNIPAM at the same $\mathrm{Na}_{2} \mathrm{SO}_{4}$ concentration.

These results suggested that thermoresponsive liquid marbles could be used to induce chemical reactions unrelated to temperature changes by floating a liquid marble containing one reactant on bulk water containing another reactant. Cooling the bulk water could lead the liquid marble to disintegrate, thereby allowing the two reactants to come into contact and triggering the reaction. This behavior was demonstrated using a liquid marble prepared from PNIPAM powder and a water droplet containing both $0.5 \mathrm{M}$ $\mathrm{Na}_{2} \mathrm{SO}_{4}$ and $0.01 \mathrm{M} \mathrm{Fe}\left(\mathrm{NO}_{3}\right)_{3}$, which was floated on the surface of bulk water containing $0.5 \mathrm{M} \mathrm{Na}_{2} \mathrm{SO}_{4}$ and $0.2 \mathrm{M} \mathrm{K}_{4}\left[\mathrm{Fe}(\mathrm{CN})_{6}\right]$ (Figure 5). $\mathrm{Fe}\left(\mathrm{NO}_{3}\right)_{3}$ and $\mathrm{K}_{4}\left[\mathrm{Fe}(\mathrm{CN})_{6}\right]$ reacted to generate water-insoluble $\mathrm{KFe}[-$ $\left.\mathrm{Fe}(\mathrm{CN})_{6}\right]$, Prussian blue. ${ }^{23}$ To verify the chemical reaction, the precipitate of Prussian blue was observed after cooling the liquid marble (Figure 5b and Supplementary Video 2 in the Supporting information).

\section{CONCLUSIONS}

This report described the preparation of thermo-responsive liquid marbles composed of PNIPAM powder and water droplets containing salts. The liquid marbles were transferred to the surface of bulk water containing salts, where they remained stable for longer than a day under humid conditions at room temperature. When the bulk aqueous solution was cooled, the liquid marbles disintegrated because the wettability of the PNIPAM powder increased. The remote triggering of chemical reactions using 
liquid marbles can be achieved by cooling. It is important to develop various external stimuli to disintegrate liquid marbles. We attempted to prepare thermo-responsive liquid marbles using powders of ureido polymers that exhibit upper critical solution temperatures in water. ${ }^{24}$ However, we could not obtain these liquid marbles because the ureido polymer powders wetted independent of temperature.

\section{ACKNOWLEDGEMENTS}

This research was partially supported by a Grantin-Aid for Scientific Research for Innovative Areas of 'Molecular Soft-Interface Science' (No. 23106717) from the Ministry of Education, Science, Sports and Culture of Japan (MEXT), and by a Grant-in-Aid for Scientific Research (B) (No. 25288101) from the Japan Society for the Promotion of Science. We also thank Kohjin for the gift of NIPAM.

Shin-ichi Yusa ${ }^{1}$, Masashi Morihara ${ }^{1}$, Keita Nakai ${ }^{1}$, Syuji Fujii ${ }^{2}$, Yoshinobu Nakamura ${ }^{2}$, Atsushi Maruyama ${ }^{3}$ and Naohiko Shimada ${ }^{3}$

${ }^{1}$ Department of Materials Science and Chemistry, University of Hyogo, Hyogo, Japan;

${ }^{2}$ Department of Applied Chemistry, Faculty of Engineering, Osaka Institute of Technology, Osaka, Japan and ${ }^{3}$ Graduate School of Bioscience and Biotechnology, Tokyo Institute of Technology, Kanagawa, Japan E-mail: yusa@eng.u-hyogo.ac.jp
1 Aussillous, P. \& Quéré, D. Liquid marbles. Nature 411, 924-927 (2001).

2 McHale, G., Elliott, S. J., Newton, M. I., Herbertson, D. L. \& Esmer, K. Levitation-free vibrated droplets: resonant oscillations of liquid marbles. Langmuir 25, 529 533 (2009).

3 Bhosale, P. S. \& Panchagnula, M. V. On synthesizing solid polyelectrolyte microspheres from evaporating liquid marbles. Langmuir 26, 10745-10749 (2010).

4 Bormashenko, E., Pogreb, R., Musin, A., Balter, R., Whyman, G. \& Aurbach, D. Interfacial and conductive properties of liquid marbles coated with carbon black. Powder Technol. 203, 529-533 (2010).

5 Matsukuma, D., Watanabe, H., Yamaguchi, H. \& Takahara, A. Preparation of low-surface-energy poly[2(perfluorooctyl)ethyl acrylate] microparticles and its application to liquid marble formation. Langmuir $\mathbf{2 7}$ 1269-1274 (2011).

6 Gao, L. \& McCarthy, T. J. Ionic liquid marbles. Langmuir 23, 10445-10447 (2007).

7 Bormashenko, E., Pogreb, R., Whyman, G., Musin, A., Bormashenko, Y. \& Barkay, Z. Shape, vibrations, and effective surface tension of water marbles. Langmuir 25, 1893-1896 (2009).

8 Bangi, U. K. H., Dhere, S. L. \& Rao, A. V. Influence of various processing parameters on water-glassbased atmospheric pressure dried aerogels for liquid marble purpose. J. Mater. Sci. 45, 2944-2951 (2010).

9 Doganci, M. D., Sesli, B. U., Erbil, H. Y., Binks, B. P. \& Salama, I. E. Liquid marbles stabilized by graphite particles from aqueous surfactant solutions. Colloids Surf. A 384, 417-426 (2011).

10 Bormashenko, E., Bormashenko, Y., Pogreb, R. \& Gendelman, O. Janus droplets: liquid marbles coated with dielectric/semiconductor particles. Langmuir $\mathbf{2 7}$ 7-10 (2011).

11 Yildirim, A., Budunoglu, H., Daglar, B., Deniz, H. \& Bayindir, M. One-pot preparation of fluorinated mesoporous silica nanoparticles for liquid marble formation and superhydrophobic surfaces. ACS Appl. Mater. Interfaces 3, 1804-1808 (2011).

12 Matsukuma, D., Watanabe, H., Minn, M., Fujimoto, A. Shinohara, T., Jinnaia, H. \& Takahara, A. Preparation of poly(lactic-acid)-particle stabilized liquid marble and the improvement of its stability by uniform shell formation through solvent vapor exposure. RSC $A d v$. 3, 7862-7866 (2013).

13 Bormashenko, E. \& Musin, A. Revealing of water surface pollution with liquid marbles. Appl. Surf. Sci. 255, 6429-6431 (2009).

14 Tian, J., Arbatan, T., Li, X. \& Shen, W. Liquid marble for gas sensing. Chem. Commun. 46, 4734-4736 (2010).

15 Arbatan, T., Li, L., Tian, J. \& Shen, W. Liquid marbles as microbioreactors for rapid blood typing. Adv. Healthcare Mater. 1, 80-83 (2012).

16 Dupin, D., Armes, S. P. \& Fujii, S. Stimulus-responsive liquid marbles. J. Am. Chem. Soc. 131, 5386-5387 (2009).

17 Fujii, S., Kameyama, S., Armes, S. P., Dupin, D. Suzaki, M. \& Nakamura, Y. pH-responsive liquid marbles stabilized with poly(2-vinylpyridine) particles. Soft Matter. 6, 635-640 (2010).

18 Zhang, L., Cha, D. \& Wang, P. Remotely controllable liquid marbles. Adv. Mater. 24, 4756-4790 (2012).

19 Nakai, K., Fujii, S., Nakamura, Y. \& Yusa, S. Ultraviolet light-responsive liquid marbles. Chem. Lett. 42, 586-588 (2013).

20 Fujishige, S., Kubota, K. \& Ando, I. Phase transition of aqueous solutions of poly( $\mathrm{N}$-isopropylacrylamide) and poly( $\mathrm{N}$-isopropylmethacrylamide). J. Phys. Chem. 93, 3311-3313 (1989).

21 Saitoh, T., Ohyama, T., Sakurai, T., Kaise, T., Takamura, K., Suzuki, Y. \& Matsubara, C. Polymer-mediated extraction of 8-quinolinolato metal chelates for the determination of metal ions in water with graphite furnace atomic absorption spectrometry. Talanta $\mathbf{4 6}$ 541-550 (1998).

22 Zhang, Y., Furyk, S., Bergbreiter, D. E. \& Cremer, P. S. Specific ion effects on the water solubility of macromolecules: PNIPAM and the Hofmeister series. J. Am. Chem. Soc. 127, 14505-14510 (2005).

23 Buser, H. J., Schwarzenbach, D., Peter, W. \& Ludi, A. The crystal structure of Prussian blue: $\mathrm{Fe}_{4}\left[\mathrm{Fe}(\mathrm{CN})_{6}\right]_{3} \cdot x \mathrm{H}_{2} \mathrm{O}$. Inorg. Chem. 16, 2704-2710 (1997).

24 Shimada, N., Ino, H., Maie, K., Nakayama, M., Kano, A. \& Maruyama, A. Ureido-derivatized polymers based on both poly(allylurea) and poly(L-citrulline) exhibit UCST-type phase transition behavior under physiologically relevant conditions. Biomacromolecules 12 3418-3422 (2011).

Supplementary Information accompanies the paper on Polymer Journal website (http://www.nature.com/pj) 